\section{Hacia un mejor mercado laboral}

Nuestro mercado del trabajo tiene muchas regulaciones inspiradas en buenas intenciones, pero muchas veces ellas perjudican a los trabajadores en vez de ayudarlos. El panorama mejoraría si los principios solidarios fueran combinados con una importante dosis de lógica económica.

Por Marcela Perticará $a^{*}$ Jorge Rodríguez Grossi**
Los temas laborales están tan intimamente vinculados con el desarrollo personal y familiar, que discutirlos solamente a partir de la racionalidad económica es, de por sí, parcial. Para construir una sociedad sin pobreza y con mayor equidad, tenemos que incorporar mecanismos de protección social frente a vicisitudes como la cesantia, el nacimiento $y$ cuidado de niños, y las enfermedades. Pero también debemos conseguir que todos los mercados, incluyendo el laboral, sean lo mas

El debate, sin embargo, está muy ideologizado, y como pais no nos hemos dado el espacio para discutir ni siquiera la posibilidad de introducir algunas reformas al mer"sado laboral. No estariamos cuestionando la "subcontratácion" si hubieramos reformado sustituyéndolo por un más potente subsidio de cesantio Quizás tampoco necesitariamos "legislar" para que las empresas pagano tuviéramos leyes que hacen a hombres mujeres tan "económicamente desiguales" frente a los empleadores.

Algunos estudios reportan que, en términos comparados, el mercado laboral chileno es altamente flexible' . Pero junto a un sector altamente protegido (asalariados privados y públicos) convive un sector informal o en ran iguales sueldos a hombres $y$ mujeres
Es decir, hay una flexibilidad efecto de la resistencia empresarial a contratos de duración indefinida altamente gravados, y un generalizado uso de esquemas de contratación al ternativos para no

tiempo indefinido.

En este articulo nos nutrimos de los anáMisis y recomendaciones del libro "Entre las bues Mejores politicas para el mercado laboral editado por Marcela Perticará, Jorge Rodriguez Grossi y Claudia Sanhueza y publicado por Ediciones Universidad Alberto Hurtado. Además de los autores mencionados en este un destacada participación en el libro son Harald Beyer, Francisca Dussaillant, Eduardo Fanizylber, Isabel Poblete, Alejendro Micco y Felipe Sáez.

1. NUDOS CRÍTICOS EN EL MERCADO LABORAL CHILENO Bajo uso de esquemas flexibles de trabajo incluso en mujeres y jóvenes, quienes son típicos trabajadores bajo esa modalidad (según la encuesta Casen 2006, más del 60\% de mujeres y jóvenes declara trabajar más de 40 horas a la semana).

En las últimas décadas se han desarrollado formas de trabajo remunerado que buscan compatibilizar las tareas familiares con vida laboral y estudio: reducción del énfasis en el tiempo presencial de trabajo, instrumentación de jornadas parciales, reducción de las semanas de trabajo en el año, horas de entrada y salidas fis sibles y teletrabajo, entre otras. Las normas sobre jornada laboral en Chile otorgan cierta fiexilidad a la empresa para organizar y distribuir esta jornada. Se tablecr jornas parciles e incluso trabaj en feriados.

Gué aspectos existe rigidez, entonces? La jornada de trabajo semanal no puede exceder las 45 horas y la jornada diaria no puede prolongarse más de diez. Se podría permitir la readecuación de las jornadas laborales a periodos estacionales, sin necesidad de recurrir a pagos por sobretiempo 0 a la externalización de ciertos servicios. Además, se podría pensar en contratos a tiempo completo que involucraran, por ejemplo, algunos dias más cortos y otros más largos,

Llion cientos dias de la semana.

Limitaciones a la negociación colectiva? Rosanna Costa reporta que la tasa de sindicamás a t que las de Estados Unidos, España más alta que las de Estados Unidos, España y Suecia Algunas encuestas sobre relaciones laborales en Chile ${ }^{2}$ reportan en generabajos niveles de conflictividad entre empleadores y empleados (89\% de los trabajadores declara tener buenas relaciones con su jefe) aunque dirigentes sindicales tienden a declaque no hay mayor participación en sindatos por miedo a represalias (45\%). Solo un $16 \%$ opina que la empresa dificulta la tarea jadores declara no estar sindica diza los trabano hay sindicato en su empresa, pero $40 \%$ dice no estar interesado en sindicalizarse Ahora bien la baja sindicalización pudier ser un efecto combinado de falta de oportunidad (los sindicatos deben tener al menos ocho miembros, por lo que la posibilidad de sindicarse en empresas pequeñas es nula pero también de falta de interés. Desde punto de vista de la empresa, en principio no hay grandes ganancias por contar con un sindicato porque hay pisos en la Ley de (las jeato de rabajo que no son negociables interlocudar, por ejemplo). Y con un unico certeria cionar la nego
limitaciones.

El costo de los contratos de tiempo indefinido vs. la aparición de relaciones contractuales vs. la aptivas. Hoy existe libertad para despeuri previo pago de un mes de preaviso y de cada año de servicio cun mes de paga por nido el trabajador, con el tope de once años. Existe plena libertad para pactar trabajos a plazo (con un tope de doce meses) o por ta rea o servicio, pero la ley establece que un contrato se transforma en indefinido si lación contractual continúa al momento de vencer el contrato o en el momento que se firma la segunda renovación de un contrato de plazo fijo. Para contratos indefinidos indemnización corresponde a todo evento, salvo cuando la causa de despido está relacionada con conductas y/o actividades dolo-

El regimen de indemnizaciones, que es un impuesto a los contratos largos, protege a los trabajadores que están contratados, pero perjudica a los nuevos y a los desempleados. Por eso no es extraño que se haya masificado el uso de contratos a plazo, trabajos a cervios la esques. Es que subcontración de proporción de trabs que mientras mayor

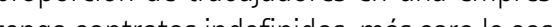
táa a ésta ajustar su dotaion carolecos(ns negativos. como tipicamente ocurre con los ciclos, 10 óptimo seria que se pudiera tombién transitoriamente, austar jornada, sin necesarimente despedir trabajidores. Pero esto está gravado porque involucra el pago de una indemnización parcial a los trabajadores plazo indefinido.

- Introducir cambios al régimen de contratos indefinidos es necesario para que la norma sea la contratación indefinida. García, Gonzallez y Navarro (2009) encuentran que una del régimen de indemnizaciones, acompañada por un aumento en el impuesto al salario. no al contrato indefinido, con el proposito de financiar mayor protección social en caso de despido, tendía claros efectos positivos so-

Jeres que searecen la contratación de muJers. Hay dos instituciones que aumentan cencis matinales contratar mujeres. las itsalas cunas que tienen las encón se proveer

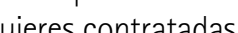

plan una licencia por maternidad de 18 semanas (seis de preparto y 12 de posparto) fuero de 12 meses luego de lo anterior, derecho a sala cuna por dos años, derecho dos medias horas diarias durante dos años para alimentar al niño y derecho a licencias especiales por enfermedad del niño menor de un año $o$ por enfermedad grave de un hijo

Las licencias maternales favorecen la lac- 


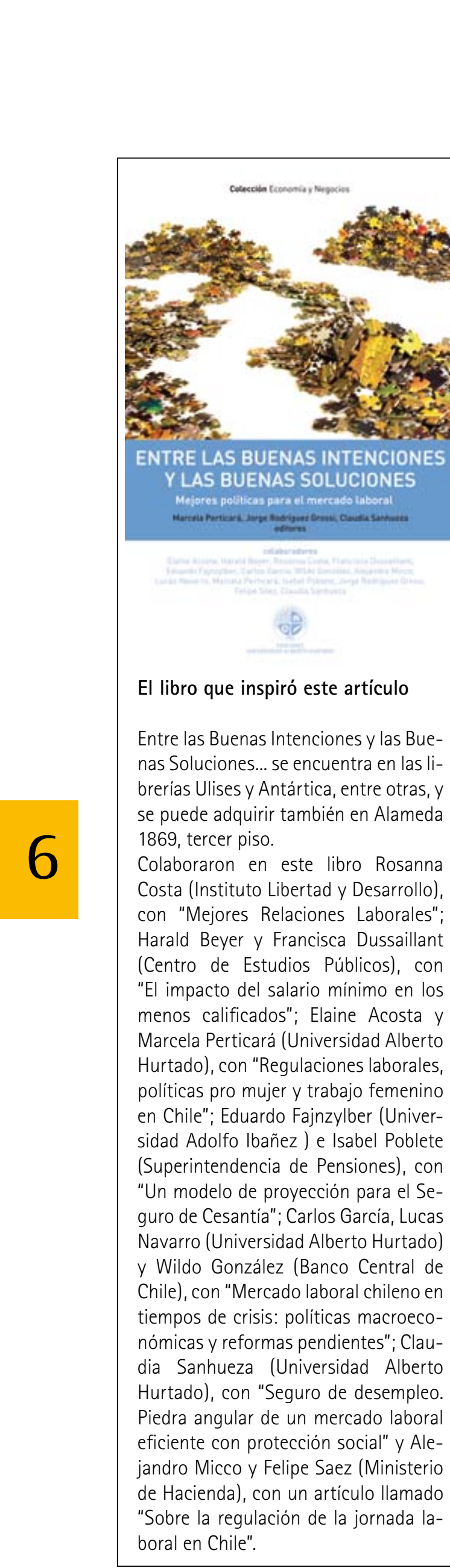

बहै

El libro que inspiró este artículo

Entre las Buenas Intenciones y las Buenas Soluciones... se encuentra en las librerias Ulises y Antártica, entre otras, y se puede adquirir también en Alameda
1869, tercer piso.

Colaboraron en este libro Rosanna Costa (Instituto Libertad y Desarrollo), con "Mejores Relaciones Laborales", (Centro de Estudios Públicos), con "El impacto del salario minimo en los menos calificados": Elaine Acosta y Marcela Perticará (Universidad Alberto Hurtado), con "Regulaciones laborales, politicas pro mujer y trabajo femenino en Chile"; Eduardo Fajnzylber (Universidad Adolfo Ibañez ) e Isabel Poblete (Superintendencia de Pensiones), con "Un modelo de proyección para el Seguro de Cesantia"; Carlos García, Lucas Navarro (Universidad Alberto Hurtado) y Wildo González (Banco Central de
Chile), con "Mercado laboral chileno en tiempos de crisis: políticas macroeconómicas y reformas pendientes"; Claudia Sanhueza (Universidad Alberto Hurtado), con "Seguro de desempleo. Piedra angular de un mercado labora eficiente con protección social" y Alejandro Micco y Felipe Saez (Ministerio de Hacienda), con un articulo llamado boral en Chile". tancia y el apego entre madre e hijo, pero re- binar beneficios de desempleo con instituducen los incentivos para contratar mujeres, ciones de intermediación laboral, y condicioespecialmente en puestos claves o sofisti- nar la entrega continua de estos beneficios cados. En Chile ni siquiera existe la posibi- que el trabajador participe en forma activa lidad de pactar con el empleador trabajo a en progras la de capacitación (si asi lo necejornadas parciales y/o flexioilización de los descansos pre y posnatal. Asi, una politica de la mujer perfectamente puede terminar afectando su inserción laboral.

Algo similar sucede con el derecho a sala cuna. Esta norma busca proteger el derecho de los niños a ser cuidados y alimentados mientras su madre trabaja. Sin embargo, la forma de elegibilidad y de financiamiento tiene efectos adversos sobre las oportunidades de empleo femenino. Este esquema desincentiva la contratación de más de 19 mujeres en empresas medianas y la de mujeres en edad fértil en empresas de mayor tamaño. Es mas: la regulación tiene una baja cobertura, pues, de acuerdo a la Dirección del Trabajo, solo 13\% de las empresas están obligadas a cumplirla. Esta norma funcion bajo la logica de que la responsabilidad de cuidado es solamente materna, pero la ella debiera también recaer en el padre o en la familia, para que disminuya el sesgo econó-
mico contra la mujer. mico contra la muje

El sistema de protección contra el desempleo. Tal como destaca Claudia Sanhueza, el sistema de seguro de cesantia en Chile preindividuales no son suficientes para fin locio los pagos a seguro, en la mayria de los casos se uniliza el Fondo de Cesantia Solidario. Además, existen topes de remuneraciones tasas de reemplazo muy bajas, que empiezan en $50 \%$ y bajan hasta 200\%. Otro punto: como el esquema de financiamiento $y$ beneficios diferencian entre trabajadores a plazo con contrato indefinido, es el mismo sistem el que replica la lógica de segmentación de mercado actual: hay un grupo de trabajadores más protegido que otro. Aún más: los beneficios por desempleo solo pueden cobrarse en la medida que el trabajador pierda en forma indefinida su trabajo. Si un trabajador ve disminuido su salario en forma transitori y necesita hacer uso de su cuenta individua no puede

La literatura enfatiza la necesidad de comrarse mediante la negociación colectiva. No

se trata de menoscabar derechos adquiridos
sino de negociar con el ánimo de aumenta
productividad y generar ganancias también

sino de negociar con el ánimo de aumentar
productividad y generar ganancias también para los trabajadores.

Flexibilizar los esquemas de jornada laboral. Los esquemas alternativos de jornadas laborales flexibles que emplean paises industrializados no deben verse como una manera rabien ales. Por el contranio, pueden consticuirse en grupos demográficos en el mercado booral (por ejemplo, jóvenes y mujeres). Podria permitirse la anualización o semestralización de la jornada laboral, para poder acomodar periodos de alta y baja demanda en ciertas actividades. 0 el uso de semanas comprimidas, con días laborales más prolongados. Por ejemplo: ciertas actividades tienen mayo demanda entre lunes y jueves, y poco movimiento durante los viernes a la tarde. Bajo un esquema flexible un trabajador pudiera optar por trabajar de lunes a jueves 10 horas por dia, y sólo trabajar los viernes hasta las $13 \mathrm{hrs}$., sin que esto obligue al pago de horas extras para la empresa. Algunos de estos esquemas pudieran negociarse por acuerdo colectivo o solo permitirse en empresas que cuenten con algún sello de certificación de buenas prácticas laborales.

Instrumentar un subsidio al trabajo a tiempo parcial. Hay que fomentar el uso de jornadas parciales para favorecer la transición con trabajo. El trabajo inclusive debería ser en horarios centrales, par compatibilizar jornada laboral con la escolar en el caso de las mujeres $y$ con estudio vespertino en caso de los jóvenes egresados de la enseñanza media que desean continuar estudios superiores. Hay una propuesta, originalmente realizada por Salvador Valdés $\left(2009^{5}\right)$ también mencionada por Claudia Sanhueza (2009), que propone el subsidiar en forma sidio va en dos direcciones. Por un lado, en la actual crisis puede potenciar la retención de mano de obra en contratos parciales. Por el otro, si el subsidio es permanente, pued incentivar el uso de jornadas parciales.

Flexibilización de las licencias maternales. Acosta y Perticará proponen transitar hacia un descanso maternal flexible y remover la obligatoriedad (con algunos pisos) de tomar periodos de pre y posnatal de duración defidirecta el trabajo a tiempo parcial. Este sub-

nida. La necesidad de proteger los derechos contexto de crisis. Pero la evidencia empiride la mujer y de sus hijos plantea la necesi- ca sugiere, lamentablemente, que subsidios dad de mantener la obligatoriedad del des- a la contratación no son efectivos para gecón de lo losivos. Los cion de la licencia (voluntaria) para mujeres beneficios de los programas de capacitación

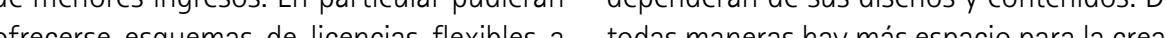
mujeres de altos ingresos, quienes en cierta tividad. Una medida que debiera estudiarse medida se ven más perjudicadas a perder in - es permitir que las empresas, frente a un gresos durante los periodos de licencia ${ }^{6}$. Hay shock negativo y generalizado de demanda aue tener en cuenta que para un empleador - puedan alterar la duración de la jornada y el costo de la licencia no es cero, ya que tiene remuneración de los trabajadores a tiemque conseguir reemplazo y, segundo, adap- po indefinido, sin que esto les signifique el tarse y capacitar a la nueva trabajadora. Es cancelar ningún tipo de indemnización. Así más complejo reemplazar trabajadores en mismo, el trabajador despedido pudiera acoposiciones de responsabilidad, por lo que li- gerse a pagos del seguro de cesantía que le cencias muy prolongadas perjudican más, en cubrieran parcialmente la pérdida de salario. términos relativos, las posibilidades de em- Si el mercado laboral tiene malas regulapleo de mujeres de mayor calificación. ciones económicas, aunque ello sea debido Suspender la obligación de las empresas de a las mejores intenciones, la economia enteproveer servicios de sala cuna, y moverse ha- ra sufrirá porque habrá un sesgo contra la colo do de cuidado infantil. Hay que transitar de con los principios de solidaridad, de protecla obligatoriedad de provisión de sala cuna a ción efectiva de la maternidad, de atención al a cesante y su familia, es una obligación etica bajadoras, a un esquema en que el cuidado que la politica debe asumir con valentia en Ta por la sociedado se manera compartizando el acceso a cuidado infantil gratuito al 40\% más pobre de la población, pero diseñar un sistema de copagos para las familias de un sistes Fortalecimiento del sistema de protección al desempleo Es necesario reforar Oficinas Municipales de Intermediación Laboral, OMLL (o su reemplazo por alguna otra institución) como ventanillas únicas para acceder a servicios de capacitación de intermediación laboral y otros programas públicos de protección al desempleado. También hay que estudiar mecanismos que permitan flexibilizar el acceso al Fondo Solidario y explazo del seguro.

Sobre mecanismos de protección al desem pleo en tiempos de crisis. En tiempos de crisis es necesario implementar politicas contraciclicas. Las últimas medidas implementadas para retener y capacitar a los trabajadores son buenas porque tratan de evitar que las (a) sabemos terminan perjudicando a la sociedad.

'Ver ALBAGLL, P. GARCiA. y J. RESTREPO. Assessing
the Flexibility of the Labor Market in Chile: An Interthe Flexibility of the Labor Market in Chile: An Inter-
national Perspective. Labor Markets and Institutions (J. Restrepo y A. Tokman, eds.), Santiago: Banco Central
de Chile, 2005. "Ver Encuesta ENCLA del Ministerio del Trabajo y encuesta realizada por el Consejo Asesor Presidencial
de Trabajo y Equidad.
${ }^{3}$ AGOSOSTINI, Claudio. Desempleo, Presiones Sectoriales ${ }^{3}$ AGOSTINI, Claudio. Desempleo, Presiones Sectoriales
y Rol del Estado. En <http://www.blogeconomia.uahurtadocl/? ps p110>.
${ }^{4}$ Según la Encuesta CASEN 2006 "Según la Encuesta CASEN 2006.
"VADESS, S. Subsidio a la conservación del empleo Puntos de Referencia. CEP Chile, 2009, p. 310 .
6 El s subsidio.

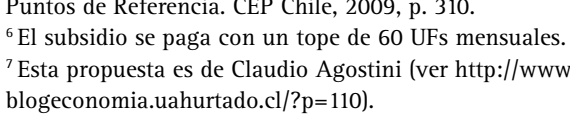

Oe

Decano: Jorge Rodríguez Grossi.
Fono Facultad: 8897366 mail: jrodrigu@uahurtado. economiaynegocios.uahurtado.cl/observato Periocion OE: Comunicar, Escuela de

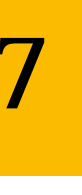

